\title{
GENERALIZATION OF THE GROUPS OF GENUS ZER0*
}

\author{
G. A. MILLER
}

One of the most important systems of groups is that which is composed of all the groups which may be defined by the orders of two generators $\left(s_{1}, s_{2}\right)$ and the order of their product. It is known that these three orders determine a finite group only when one of them is unity, when two of them are equal to 2 , or when one is 2 while the other two are one of the following three pairs of numbers : 3,$3 ; 3,4 ; 3,5 . \dagger$ The first of these sets of orders define a cyclic group and may be regarded as trivial. When the order of two among the three operators $s_{1}, s_{2}, s_{1} s_{2}$ is equal to 2, the group $\left\{s_{1}, s_{2}\right\}$, generated by $s_{1}, s_{2}$, is the dihedral rotation group whose order is twice that of the third operator. This useful category has recently been generalized under the heading, The groups generated by two operators which have a common square. $\neq$ This generalized category could also be defined as the set of groups generated by two operators such that the square of one of them is equal to the square of their product.

The object of the present paper is to complete this kind of generalization for the dihedral rotation group and to extend it to the other groups of genus zero. The resulting groups have a two-fold interest in view of their close contact with the important system of groups of genus zero and their elementary structures. It is believed that a complete list of these groups will prove very useful in many investigations. Our object is to study all the groups which result when one of the three conditions which may be used to define a group of genus zero is preserved while the other two are replaced by a single one of an elementary type. For instance, the dihedral rotation groups are defined by the conditions $\S$

$$
s_{1}^{2}=1, \quad s_{2}^{2}=1, \quad\left(s_{1} s_{2}^{-1}\right)^{n}=1 .
$$

In the generalization mentioned above these conditions are replaced by

\footnotetext{
* Presented to the Society at the New Haven meeting September 3, 1906. Received for publication July 11, 1906.

† American Journal of Mathematics, vol. 24 (1902), p. 96.

¥ Arohiv der Mathematik und Physik, vol. 9 (1905), p. 6.

$\S$ Throughout the paper care has been taken to insure that, when one of the given generational relations of a group is $8^{n}=1$, the operator 8 shall be of order $n$.
} 


$$
s_{1}^{2}=s_{2}^{2}, \quad\left(s_{1} s_{2}^{-1}\right)^{n}=1 .
$$

A similar generalization is given by the equations

$$
s_{1}^{2}=s_{2}^{n}, \quad\left(s_{1} s_{2}^{-1}\right)^{2}=1 \quad(n>2) .
$$

We proceed to consider the groups whose generators satisfy the last equation. From $\left(s_{1} s_{2}^{-1}\right)^{2}=1$ it results that $s_{1} s_{2}^{-1} s_{1}=s_{2}$. Hence $s_{1}^{-1} s_{2} s_{1}=s_{2}^{n-1}$ and $s_{1}^{-1} s_{2}^{n} s_{1}=s_{2}^{n^{2}-n}$ Since $s_{2}^{n}$ is invariant under $\left\{s_{1}, s_{2}\right\}$ it is necessary that $n^{2}-n \equiv n(\bmod m)$, $m$ being the order of $s_{2}$. That is, $m$ must divide $(n-1)^{2}-1$. Since $\left\{s_{1}, s_{2}\right\}$ is generated by $s_{2}$ and $s_{1} s_{2}^{-1}$ and since the latter transforms the former into its $(n-1)$-th power, the order of $\left\{s_{1}, s_{2}\right\}$ is $2 m$. These results may be expressed as follows: If the two generators of a group satisfy the conditions $s_{1}^{2}=s_{2}^{n}$, $\left(s_{1} s_{2}^{-1}\right)^{2}=1, n>2$, it is non-abelian, involves a cyclic subgroup of half its order, and an additional operator of order 2 which transforms each operator of this cyclic subgroup into its $(n-1)$-th power.

For $m=n$ this category of groups reduces to the dihedral rotation groups. It is clear that we may assume that $m \geqq n$; for, if $m$ were less than $n$, the number $n$ could be replaced by $n-k m$, where $k$ is arbitrary. The invariant operators of $\left\{s_{1}, s_{2}\right\}$ constitute a subgroup whose order is the highest common factor of $m$ and $n-2$. With respect to this subgroup the quotient group of $\left\{s_{1}, s_{2}\right\}$ is a dihedral rotation group. If $H$ represents any Sylow subgroup of odd order in $\left\{s_{1}, s_{2}\right\}$, then either $s_{1}$ is commutative with each operator of $H$ or it transforms each of these operators into its inverse. In the former case the order of $s_{1}$ is divisible by that of $H$. In the latter case the orders of $s_{1}$ and $H$ are relatively prime. The numbers $n$ and $m$ cannot be relatively prime since $\left\{s_{1}, s_{2}\right\}$ is non-cyclic. For the same reason $s_{1}$ must be of even order, as is also otherwise evident.

It may be added that if $n$ is replaced by $n^{\prime}=m-n$, the given equations become

$$
t_{1}^{2}=t_{2}^{n^{\prime}}, \quad\left(t_{1} t_{2}\right)^{2}=1 \quad\left(n^{\prime}<m-2\right) .
$$

Hence these equations define the same system of groups as those given above. Similar remarks apply to the system defined by the equations $t_{1}^{2}=t_{2}^{2},\left(t_{1} t_{2}\right)^{n}=1$. Since the dihedral rotation groups constitute an infinite system it is necessary that the generalization of the conditions satisfied by its generators are also satisfied by the generators of an infinite system of groups. The other groups of genus zero are defined as individuals, and the corresponding generalizations are satisfied by a very small number of groups.

\section{§1. Generalization of the tetrahedral group.}

It is known that any two operators of order 3 whose product is of order 2 
generate the tetrahedral group. That is, this group is completely defined by two operators $s_{1}, s_{2}$ which satisfy either of the following sets of conditions :

$$
s_{1}^{3}=s_{2}^{3}=1,\left(s_{1} s_{2}\right)^{2}=1 ; \quad s_{1}^{2}=s_{2}^{3}=1,\left(s_{1} s_{2}\right)^{3}=1 .
$$

We proceed to consider the groups which satisfy the more general equations

$$
s_{1}^{3}=s_{2}^{3},\left(s_{1} s_{2}\right)^{2}=1 ; \quad s_{1}^{2}=s_{2}^{3},\left(s_{1} s_{2}\right)^{3}=1 .
$$

We begin with the first of these two cases; that is, we consider all the groups which are generated by two operators having a common cube and a product of order 2.

The three operators $s_{1} s_{2}, s_{2} s_{1}, s_{1}^{-1} s_{2} s_{1}^{2}$ are conjugate under $s_{1}$ and the product of the last two is $s_{2}^{2} s_{1}^{2}=s_{1} s_{2} \cdot s_{1}^{6}$ since $s_{1} s_{2}=s_{2}^{-1} s_{1}^{-1}$. As $s_{2} s_{1}$ transforms this product into its inverse and is commutative with $s_{1}^{6}$ the order of $s_{1}$ must divide 24. As it is a multiple of 3 it has one of the three valuees $24,12,6,3$. If this order is 24 the two operators of order two $s_{2} s_{1}, s_{1}^{-1} s_{2} s_{1}^{2}$ generate the octic group since $s_{2}^{2} s_{1}^{2}$ is of order 4 . This octic group and $s_{1}^{6}$ generate an invariant subgroup of order 16 since it is transformed into itself by $s_{1}$ and also by $s_{2}$. As this invariant subgroup contains the quaternion group as a characteristic subgroup, this quaternion group and $s_{1}^{8}$ generate the group of order 24 which does not involve a subgroup of order 12. Hence the given group of order 16 and $s_{1}^{8}$ generate the group of order 48 known as $G_{50} \cdot{ }^{*}$ As this involves $s_{1}^{6}$ and $s_{1}^{8}$ it also involves $s_{1}^{2}$. If we extend it by means of $s_{1}^{3}$ there results a group of order 96 which involves both $s_{1}$ and $s_{2}$ and hence is $\left\{s_{1}, s_{2}\right\}$. As $s_{1}^{3}$ is invariant under $\left\{s_{1}, s_{2}\right\}$ there is only one such group. $\dagger$ It may be represented as a substitution group of degree 32 by means of

$$
\begin{aligned}
& s_{1}=a c^{\prime} c e^{\prime} e g^{\prime} g a^{\prime} \cdot b m^{\prime} n f^{\prime} o j^{\prime} h k^{\prime} l d^{\prime} m p^{\prime} f^{\prime} j b^{\prime} k n^{\prime} d o^{\prime} p h^{\prime} i l^{\prime}, \\
& s_{2}=a m^{\prime} j e^{\prime} o n^{\prime} g k^{\prime} p c^{\prime} m l^{\prime} e i^{\prime} n a^{\prime} k j^{\prime} c o^{\prime} l g^{\prime} i p^{\prime} \cdot b d^{\prime} d f^{\prime} f h^{\prime} h b^{\prime} .
\end{aligned}
$$

When $s_{1}$ is of order 12 the two operators $s_{2} s_{1}, s_{1}^{-1} s_{2} s_{1}^{2}$ are commutative since their product is of order 2. Hence $\left\{s_{2} s_{1}, s_{1}^{-1} s_{2} s_{1}^{2}\right\}$ is the four-group. This four-group and $s_{1}^{6}$ generate the group of order 8 which involves seven operators of order 2. This is invariant under $\left\{s_{1}, s_{2}\right\}$. Since the group of isomorphisms of this group of order 8 is known, it is known that $s_{1}^{4}$ and one of its subgroups of order 4 generate the tetrahedral group. Hence $\left\{s_{1}, s_{2}\right\}$ is the direct product of this tetrahedral group and $s_{1}^{3}$. When $s_{1}$ is of order 6 the four-group $\left\{s_{2} s_{1}, s_{1}^{-1} s_{2} s_{1}^{2}\right\}$ involves $s_{1} s_{2}$, and together with $s_{1}^{2}$ generates the tetrahedral group. Hence $\left\{s_{1}, s_{2}\right\}$, in this case, is the direct product of the tetrahedral group and the group of order 2 . These results may be expressed as follows :

* Quarterly Journal of Mathematios, vol. 30 (1898), p. 258.

†Balletin of the American Mathematical Society, vol. 3 (1897), p. 218. 
There are exactly four groups whose two generators $s_{1}, s_{2}$ satisfy the relations $s_{1}^{3}=s_{2}^{3},\left(s_{1} s_{2}\right)^{2}=1$. They are the tetrahedral group; the direct products of the tetrahedrl group and the cyclic group of order 4 or the group of order 2 ; and the group of order 96 considered above.

The second generalization of the tetrahedral group relates to the groups whose two generators satisfy the equations

$$
s_{1}^{2}=s_{2}^{3}, \quad\left(s_{1} s_{2}\right)^{3}=1
$$

The three conjugates of $s_{1}$ under $s_{2}$ are

$$
s_{1}, \quad s_{2}^{-1} s_{1} s_{2}, \quad s_{2}^{-2} s_{1} s_{2}^{2} .
$$

As these conjugates have a common square we are concerned with a generalization of the dihedral rotation group. Multiplying the second into the inverse of the third, we get

$$
s_{2}^{-1} s_{1} s_{2} \cdot s_{2}^{-2} s_{1}^{-1} s_{2}^{2}=s_{2}^{-1} s_{1} s_{2}^{-1} s_{1} s_{2}^{2} \cdot s_{1}^{-2}=s_{1}^{5} .
$$

Since $s_{1}^{5}$ is transformed into its inverse by $s_{2}^{-1} s_{1} s_{2}$ and $s_{1}^{2}$ is invariant under $\left\{s_{1}, s_{2}\right\}$ it follows that the order of $s_{1}$ divides 20. If the order of $s_{1}$ is 20 we consider the group generated by $s_{1}^{25}=t_{1}, s_{2}^{25}=t_{2}$. From the fact that $\left(s_{1}^{25} s_{2}^{25}\right)^{3}=s_{1}^{120}\left(s_{1} s_{2}\right)^{3}=1$, it results that $t_{1}^{2}=t_{2}^{3}$ and $\left(t_{1} t_{2}\right)^{3}=1$. Hence $\left\{t_{1}, t_{2}^{-1} t_{1} t_{2}, t_{2}^{-2} t_{1} t_{2}^{2}\right\}$ is the quaternion group, which is invariant under $\left\{t_{1}, t_{2}\right\}$ since it includes $t_{1}$ and is transformed into itself by $t_{2}$. This quaternion group and $t_{2}$ generate the group of order 24 which does not contain a subgroup of order 12. Hence this is $\left\{t_{1}, t_{2}\right\}$, while $\left\{s_{1}, s_{2}\right\}$ is the direct product of $\left\{t_{1}, t_{2}\right\}$ and the cyclic group of order 5 generated by $s_{1}^{4}$.

When the order of $s_{1}$ is 10 , it follows from the preceding paragraph that $\left\{s_{1}^{25}, s_{2}^{25}\right\}$ is the tetrahedral group and that $\left\{s_{1}, s_{2}\right\}$ is the direct product of this group and the cyclic group of order 5 . These results may be expressed as follows: There are exactly four groups whose two generators satisfy the conditions $s_{1}^{2}=s_{2}^{3},\left(s_{1} s_{2}\right)^{3}=1$. They are the tetrahedral group, the group of order 24 which does not contain a subgroup of order 12, and the direct products of these groups and the cyclic group of order 5 . As only one of these groups occurs among the four given above we have seven distinct groups resulting from one or the other of the given generalizations of the defining equations of the tetrahedral group.

It may be of interest to note how a slight change in these generalizations may affect the system. Instead of the equations $s_{1}^{3}=s_{2}^{3},\left(s_{1} s_{2}\right)^{2}=1$, we proceed to consider $s_{1}^{3}=s_{2}^{3},\left(s_{1} s_{2}^{-1}\right)^{2}=1$. The three conjugates $s_{1} s_{2}^{-1}, s_{2}^{-1} s_{1}, s_{1}^{-1} s_{2}^{-1} s_{1}^{2}$ are commutative and constitute, together with the identity, the four-group since $s_{2}^{-2} s_{1}^{2}=\left(s_{1} s_{2}^{-1}\right)^{-1}=s_{1} s_{2}^{-1}$. This four-group is invariant under $\left\{s_{1}, s_{2}\right\}$ and has 
only the identity in common with the cyclic group generated by $s_{1}^{3}$. Hence the theorem :

Two operators $s_{1}, s_{2}$ which satisfy the conditions $s_{1}^{3}=s_{2}^{3}$ and $\left(s_{1} s_{2}^{-1}\right)^{2}=1$ generate the group which may be obtained by establishing $a(4, h)$ isomorphism between the tetrahedral group and a cyclic group of order $3 h$, where $3 h$ is the order of $s_{1}$.

Since there is one and only one such group for every value of $h$, a group is completely defined by the value of $h$ and the given equations. In particular, when $h=1,\left\{s_{1}, s_{2}\right\}$ is the tetrahedral group. When $h$ is prime to $3,\left\{s_{1}, s_{2}\right\}$ is the direct product of the tetrahedral group and the cyclic group of order $h . \dagger$

Similarly, the second set of equations given at the beginning of this section may be replaced by $s_{1}^{3}=s_{2}^{2}$ and $\left(s_{1}^{-1} s_{2}\right)^{3}=1$. The three conjugates of $s_{2}$ under $s_{1}$ are

$$
s_{2}, s_{1}^{-1} s_{2} s_{1}, s_{1}^{-2} s_{2} s_{1}^{2} .
$$

The product of the second into the inverse of the third is $s_{1}^{-1} s_{2} s_{1}^{-1} s_{1}^{2}=s_{2}^{-1}$. Since $s_{1}^{-1} s_{2} s_{1}$ transforms $s_{2}$ into its inverse and is commutative with $s_{2}^{2}$ the order of $s_{2}$ is either 2 or 4 . In the former case $\left\{s_{1}, s_{2}\right\}$ is the tetrahedral group. In the latter case, $\left\{\boldsymbol{s}_{2}, s_{1}^{-1} s_{2} s_{1}, s_{1}^{-2} s_{2} s_{1}^{2}\right\}$ is the quaternion group. As this is invariant under $\left\{s_{1}, s_{2}\right\}$ we have the result: If the two generators of a group satisfy the conditions $s_{1}^{3}=s_{2}^{2},\left(s_{1}^{-1} s_{2}\right)^{3}=1$, it is either the tetrahedral group or the group of order 24 which does not contain a subgroup of the order 12.

\section{§ 2. Generalization of the octahedral group.}

The octahedral group is completely defined by the fact that it may be generated by two operators of orders 2 and 4, respectively, such that their product is of order 3. That is, $s_{1}, s_{2}$ define the octahedral group provided they satisfy the equations $s_{1}^{2}=s_{2}^{4}=1,\left(s_{1} s_{2}\right)^{3}=1$, and the orders of $s_{1}, s_{2}$ are 2 and 4 respectively. We shall now consider the generalization of the octahedral group given by the equations $s_{1}^{2}=s_{2}^{4},\left(s_{1} s_{2}\right)^{3}=1$.

It will be convenient to consider the three conjugates of $s_{2}^{2}$ under $s_{1} s_{2}$. These are

$$
s_{2}^{2}, \quad s_{2}^{-1} s_{1}^{-1} s_{2}^{2} s_{1} s_{2}, \quad s_{1} s_{2} s_{2}^{2} s_{2}^{-1} s_{1}^{-1}=s_{1} s_{2}^{2} s_{1}^{-1} .
$$

The form of the last one of these three conjugates results from the fact that $\left(s_{1} s_{2}\right)^{-2}=s_{1} s_{2}$ since $\left(s_{1} s_{2}\right)^{3}=1$. These conjugates have a common square since $s_{2}^{4}$ is invariant under $\left\{s_{1}, s_{2}\right\}$. The group generated by any two of them is therefore a generalized dihedral rotation group. We shall consider the group

\footnotetext{
* Bulletin of the American Mathematical Society, vol. 1 (1897), p. 218 ; cf.
} Manning, Transactions of the American Mathematical Society, vol. 7 (1906), p. 233.

†Transactions of the American Mathematical Society, vol. 1 (1900), p. 67. 
generated by the last two. The product of the third into the inverse of the second is $s_{1} s_{2} s_{2}^{2} s_{1} s_{2} s_{2}^{-2} s_{1} s_{2}=s_{1} s_{2}^{-1} s_{1} s_{2}^{-1} s_{1} s_{2}^{5}=s_{2} s_{1}^{5} s_{1} s_{2}^{5}=s_{2}^{18}$; for, from the given conditions we have $\left(s_{2}^{-1} s_{1}^{-1}\right)^{3}=\left(s_{1}^{-1} s_{2}^{-1}\right)^{3}=1$, and therefore

$$
\left(s_{1} s_{2}^{-1}\right)^{3}=\left(s_{1}^{2} s_{1}^{-1} s_{2}^{-1}\right)^{3}=s_{1}^{6} \quad \text { or } \quad\left(s_{1} s_{2}^{-1}\right)^{2}=s_{2} s_{1}^{5} \text {. }
$$

Since $s_{1} s_{2}^{2} s_{1}^{-1}$ transforms $s_{2}^{18}$ into its inverse and has a common square with $s_{2}^{2}$, the order of $s_{2}$ is a divisor of 72. The group generated by $s_{2}^{-1} s_{1}^{-1} s_{2}^{2} s_{1} s_{2}$ and $s_{1} s_{2}^{2} s_{1}^{-1}$ contains $s_{2}^{2}$ since it contains both $s_{2}^{4}$ and $s_{2}^{18}$. This group is transformed into itself by $s_{2}$, since this operator transforms the latter generator into the former, as $s_{1} s_{2}^{2} s_{1}^{-1}=s_{1}^{-1} s_{2}^{2} s_{1}$, and also transforms their product into itself. Hence it is also transformed into itself by $s_{1}$, as it is transformed into itself by $8_{1} s_{2}$. That is, $s_{2}^{2}, s_{2}^{-1} s_{1}^{-1} s_{2}^{2} s_{1} s_{2}, s_{1} s_{2}^{2} s_{1}^{-1}$ generate a subgroup which is invariant under $\left\{s_{1}, s_{2}\right\}$. When this subgroup is extended by means of $s_{1} s_{2}$ there results another invariant subgroup since $s_{2} s_{1}=\left(s_{1}^{-1} s_{2}^{-1}\right)^{-1}, s_{1} s_{2}=s_{1}^{2} s_{1}^{-1} s_{2}^{-1} s_{2}^{2}$, and $s_{1}^{2}, s_{2}^{2}$ are in the preceding invariant subgroup. The order of $\left\{s_{1}, s_{2}\right\}$ must therefore divide the product obtained by multiplying the order of $\left\{s_{2}^{-1} s_{1}^{-1} s_{2}^{2} s_{1} s_{2}, s_{1} s_{2}^{2} s_{1}^{-1}\right\}$ by 6 . That is, this order divides $6 \cdot 4 \cdot 18=432$.

If the order of $s_{2}$ is 72, the group generated by $s_{2}^{2}, s_{2}^{-1} s^{-1} s_{2}^{2} s_{1} s_{2}, s_{1} s_{2}^{2} s_{1}^{-1}$ is of order 72 , and its invariant operators constitute the cyclice roup of order 18 . It may be defined by the fact that it is non-abelian and contains more than one cyclic subgroup of order 36 . Its three cyclic subgroups of order 36 are conjugate under $s_{1} s_{2}$ and its cyclic subgroup of order 18 is composed of the invariant operators of $\left\{s_{1}, s_{2}\right\}$. By extending this group of order 72 by means of $s_{1} s_{2}$ there results the direct product of the cyclic group of order 9 and the group of order 24 which does not contain a subgroup of order 12. As this diret product contains just three subgroups of order 24 , one of them is transformed into itself by $s_{2}$ and includes $s_{2}^{18}$. This subgroup and $s_{2}^{9}$ generate a group of order 48 , known* as $G_{52}$, and hence $\left\{s_{1}, s_{2}\right\}$ is the direct product of the latter and the cyclic group of order 9 . That is, if $s_{1}, s_{2}$ satisfy the conditions $s_{1}^{2}=s_{2}^{4}$, $\left(s_{1} s_{2}\right)^{3}=1$, then either they generate the direct product of the cyclic group of order 9 and the group of order 48 known as $G_{52}$ or they generate a quotient group of this direct product.

If the order of $s_{2}$ is 36 , the three operators $s_{2}^{2}, s_{2}^{-1} s_{1}^{-1} s_{2}^{2} s_{1} s_{2}, s_{1} s_{2}^{2} s_{1}^{-1}$ generate a group of order 36. As this is abelian and contains three cyclic subgroups of order 18 it is the direct product of the four-group and the cyclic group of order 9. By extending it by means of $s_{1} s_{2}$ we obtain the direct product of the alternating group of order 12 and the cyclic group of order 9 . Hence $\left\{s_{1}, s_{2}\right\}$ is the direct product of the symmetric group of order 24 and the cyclic group of order 9 whenever the order of $s_{2}$ is 36 . If the order of $s_{2}$ were not divisible by 4 ,

* Quarterly Journal of Mathematics, vol. 30 (1898), p. 258. 
$s_{2}^{4}$ would be equal to the square of a generator of $s_{2}$ and hence we would be dealing with a group generated by two operators having a common square. Hence it is only necessary to consider the cases when the order of $\boldsymbol{s}_{\mathbf{2}}$ has one of the following values: $4,12,8,24$. In the first two cases, we arrive at the symmetric group of order 24 , or the direct product of this and the group of order 3. In the last two cases we arrive at the group of order 48 considered in the preceding paragraph or the direct product of this and the group of order 3. As the considerations are similar to those given above, it seems unnecessary to enter into details, especially since the quotient groups mentioned at the end of the preceding paragraph are so well known from the fact that $G_{52}$ has a $(2,1)$ isomorphism with the symmetric group of order 24 .

A similar generalization of the definition of the octahedral group is given by the equations

$$
s_{1}^{2}=s_{2}^{3}, \quad\left(s_{1} s_{2}\right)^{4}=1 .
$$

To find an upper limit for the order of $s_{2}$ we may consider the following product of two conjugate operators of order 2 :

$$
\left(s_{2} s_{1} s_{2} s_{1} \cdot s_{1} s_{2} s_{1} s_{2}\right)^{2}=\left(s_{1}^{2} s_{2} s_{1} s_{2}^{2} s_{1} s_{2}\right)^{2}=s_{1}^{4} s_{2} s_{1} s_{2}^{2} s_{1} s_{2}^{2} s_{1} s_{2}^{2} s_{1} s_{2}^{2} s_{2}^{-1}=s_{2}^{30} .
$$

As $s_{2}^{30}$ is both commutative with $\left(s_{2} s_{1}\right)^{2}$ and transformed into its inverse by this operator, its order cannot exceed 2 and hence the order of $s_{2}$ divides 60 . We shall first consider the group generated by $s_{1}^{5}, s_{2}^{5}$ when the order of $s$ is 60 . It is clear that these two operators satisfy the given conditions since

$$
\left(s_{1}^{5} s_{2}^{5}\right)^{4}=\left(s_{1}^{4} s_{2}^{3} s_{1} s_{2}^{2}\right)^{4}=s_{1}^{16} s_{2}^{12}\left(s_{1} s_{2}^{2}\right)^{4}=s_{2}^{60} .
$$

It will appear that $\left\{s_{1}, s_{2}\right\}$ is the direct product of $\left\{s_{1}^{5}, s_{2}^{5}\right\}$ and the cyclic group generated by $s_{2}^{12}$. From the equation given above it follows that $\left\{s_{2} s_{1} s_{2} s_{1}, s_{1} s_{2} s_{1} s_{2}\right\}$ is the octic group. Extending this by means of $s_{2}^{15}$, we obtain a group of order 16 which is invariant under $\left\{s_{1}, s_{2}\right\}$ since $s_{2}$ transforms one generator of this octic group into the other, and the product of the two into $s_{1}^{3} s_{2}^{2} s_{1} s_{2}^{2}=\left(s_{2} s_{1} s_{2} s_{1}\right)^{-1} \cdot s_{2}^{15}$. By extending this group of order 16 by means of $s_{2}^{5}$, we get a group of order 48 which is invariant under $\left\{s_{1}, s_{2}\right\}$ since $s_{1}^{-1} s_{2}^{5} s_{1}=\left(s_{2} s_{1} s_{2} s_{1}\right)^{-1} \cdot s_{2}^{10}$. Finally, if we extend this by means of $s_{1}^{3}$ we obtain the group of order 96 in question. It is easy to verify that this group may be generated by the following substitutions :

$$
\begin{aligned}
& s_{1}=a a^{\prime} g g^{\prime} e e^{\prime} c c^{\prime} \cdot b o^{\prime} h m^{\prime} f k^{\prime} d i^{\prime} \cdot i d^{\prime} o b^{\prime} m h^{\prime} k f^{\prime} \cdot j n^{\prime} p l^{\prime} n j^{\prime} l p^{\prime}, \\
& s_{2}=a m l g k j e i p c o n \cdot b d f h \cdot a^{\prime} m^{\prime} l^{\prime} g^{\prime} k^{\prime} j^{\prime} e^{\prime} i^{\prime} p^{\prime} c^{\prime} o^{\prime} n^{\prime} \cdot b^{\prime} d^{\prime} f^{\prime} h^{\prime} .
\end{aligned}
$$

When the order of $s_{2}$ is 60 the invariant subgroup generated by $s_{2}^{12}$ has only the identity in common with the $G_{96}$ of the preceding paragraph. Hence 
$\left\{s_{2}^{12}, s_{1}^{5}, s_{2}^{5}\right\} \equiv\left\{s_{1}, s_{2}\right\}$ is the direct product of $G_{96}$ and the cyclic group of order 5. When the order of $s_{2}$ is 12 it follows directly from the preceding paragraph that $\left\{s_{1}, s_{2}\right\}$ is $G_{96}$. Hence it remains to consider the cases when the order of $s_{2}$ is not divisible by 4 . If this order is 30 we again consider $\left\{s_{1}^{5}, s_{2}^{5}\right\}$. Hence $\left\{s_{2} s_{1} s_{2} s_{1}, s_{1} s_{2} s_{1} s_{2}\right\}$ is either the four-group or the group of order 2. In the former case it does not contain $s_{2}^{15}$, since one of its two generators is transformed into the other by $s_{2}$ and their product is transformed into itself multiplied by $s_{2}^{15}$ by the same operator. Hence this four-group and $s_{2}^{15}$ generate the group of order 8 which contains 7 operators of order 2 . Just as in the preceding paragraph, we observe that this group of order 8 is invariant under $\left\{s_{1}, s_{2}\right\}$.

As $s_{2}^{5}$ transforms this group of order 8 into itself and is commutative with only one of its operators of order 2 , it transforms one of its subgroups of order 4 into itself and this subgroup and $s_{2}^{10}$ generate the alternating group of order 12.* That is, $s_{2}^{5}$ and this group of order 8 generate the direct product of the alternating group of order 12 and an operator of order 2. By extending this group of order 24 by $s_{1}^{5}$ we obtain the group of order 48 which has 24 additional operators of order 4 of which 12 have the same square as $s_{1}^{5}$ while the other 12 have a different common square. This elementary group of order 48 is $\left\{s_{1}^{5}, s_{2}^{5}\right\}$ and has the symmetric group of order 24 for its group of cogredient isomorphisms. When $s_{2}$ is of order $6,\left\{s_{1}, s_{2}\right\}$ is this group of order 48 , and when $s_{2}$ is of order 30 it is the direct product of this group and the cyclic group of order 5 .

It remains only to consider the cases when $s_{2}$ is of order 3 or 15 . In the former case $\left\{s_{1}, s_{2}\right\}$ is clearly the octahedron group, while in the latter it is the direct product of this and the cyclic group of order 5 , since $s_{1}^{5}, s_{2}^{5}$ generate the octahedron group. The proof of this fact follows directly from the preceding paragraphs. Hence there are just six groups whose two generators satisfy the conditions $s_{1}^{2}=s_{2}^{3},\left(s_{1} s_{2}\right)^{4}=1$. Three of these are of orders 24,48 and 96 , respectively; while the other three are the direct products of these groups and the cyclic group of order 5 .

It remains to consider the generalization of the octahedral group whose two generators satisfy the equations

$$
s_{1}^{3}=s_{2}^{4}, \quad\left(s_{1} s_{2}\right)^{2}=1 .
$$

The superior limit of the order of $s_{2}$ may be found as follows :

since

$$
\left(s_{2}^{-2} s_{1}^{-2} s_{2}^{2} s_{1}^{2}\right)^{2}=s_{2}^{-16}\left(s_{2}^{2} s_{1} s_{2}^{2} s_{1}^{2}\right)^{2}=s_{2}^{-24}\left(s_{2} s_{1}^{2}\right)^{4}=s_{2}^{-28},
$$

$$
s_{1} s_{2}^{2}=s_{2}^{-1} s_{1}^{-1} s_{2}, \quad\left(s_{2} s_{1}^{2}\right)^{n}=s_{1}^{-1} s_{2}^{-n} s_{1} .
$$

* Cf. Moore, Bulletin of the American Mathematical Sooiety, vol. 1 (1894), p. 61. 
As $s_{2}^{-2}$ and $s_{1}^{-2} s_{2}^{2} s_{1}^{2}$ have a common square, $s_{2}^{-2 s}$ is transformed into its inverse and into itself by $s_{2}^{2}$. That is, the order of $s_{2}$ divides 56 . The two operators $s_{1}^{49}, s_{2}^{49}$ also satisfy the given conditions since $\left(s_{1}^{49} s_{2}^{49}\right)^{2}=s_{2}^{224}\left(s_{1} s_{2}\right)^{2}=1$. If the order of $s_{2}$ is 56 the orders of these two operators are 6 and 8 respectively. We proceed to consider the group generated by them.

From the given equations it follows that $s_{2}^{98}, s_{1}^{-98} s_{2}^{98} s_{1}^{98}$ generate the quaternion group. That this is invariant under $\left\{s_{1}, s_{2}\right\}$ follows from the proof that $\left\{s_{2}^{2}, s_{1}^{-2} s_{2}^{2} s_{1}^{2}\right\}$ is invariant under $\left\{s_{1}, s_{2}\right\}$. That this group contains

$s_{1}^{-1} s_{2}^{2} s_{1}=s_{2}^{-4} \cdot s_{1}^{2} s_{2}^{2} s_{1}=s_{2}^{-4} s_{1} s_{2}^{-1} s_{1}^{-1} s_{2} s_{1}=s_{2}^{-4} s_{1} s_{2}^{-1} s_{1}^{-2} s_{2}^{-1}=s_{2}^{-8} s_{1} s_{2}^{-2} s_{1}^{-1} s_{2}^{-2}=s_{2}^{-20} s_{1} s_{2}^{2} s_{1}^{2} s_{2}^{2}$

follows from the given form of the product $s_{2}^{-2} \cdot s_{1}^{-2} s_{2}^{2} s_{1}^{2}$. It is transformed into itself by $s_{2}$ since $s^{2}$ transforms $s_{2}^{2} s_{1} s_{2}^{2} s_{1}^{2}$ into $s_{2} s_{1} s_{2}^{2} s_{1}^{2} s_{2}=s_{1}^{3} \cdot s_{1}^{-1} s_{2}^{2} s_{1} s_{2}^{2}$, which is in this group. The quaternion group $\left\{s_{2}^{98}, s_{1}^{-98} s_{2}^{98} s_{1}^{98}\right\}$ and $s_{1}^{49}$ generate the group of order 24 which does not contain a subgroup of order 12. As this is clearly invariant under $\left\{s_{1}, s_{2}\right\}$ it results that $\left\{s_{1}^{49}, s_{2}^{49}\right\}$ is the group of order 48 known* as $G_{51}$, which is very closely related to the group of this order $G_{52}$ considered at the beginning of this section. When the order of $s_{2}$ is $8,\left\{s_{1}, s_{2}\right\}$ is therefore $G_{51}$, and when this order is $56,\left\{s_{1}, s_{2}\right\}$ is the direct product of $G_{51}$ and the cyclic group of order 7 , since the group generated by $s_{2}^{8}$ is invariant under $\left\{s_{1}^{49}, s_{2}^{49}\right\}$ and has only the identity in common with it.

When the order of $s_{2}$ is $28,\left\{s_{1}^{49}, s_{2}^{49}\right\}$ is the symmetric group of order 24 and $\left\{s_{1}, s_{2}\right\}$ is the direct product of it and the cyclic group of order 7 . Hence there are exactly four groups whose two generating operators satisfy the conditions $s_{1}^{3}=s_{2}^{4},\left(s_{1} s_{2}\right)^{2}=1$. Two of these are of orders 24 and 48 respectively while the other two are the direct products of these and the cyclic group of order 7. It may be added that the three groups of order 48 which have been considered in this section are distinct.

\section{§3. Generalization of the icosahedral group.}

If two operators $s_{1}, s_{2}$ satisfy the equations

$$
s_{1}^{2}=1, \quad s_{2}^{5}=1, \quad\left(s_{1} s_{2}\right)^{3}=1,
$$

they generate the simple group of order 60 . This fact was observed by HaMiLTON as early as $1856+$, and it is of especial interest since there are only a few finite groups which may be defined in such an elementary manner. It is well known that the 60 operators of this group may be written as follows:

$$
s_{2}^{m}, s_{2}^{m} s_{1} s_{2}^{n}, s_{2}^{m} s_{1} s_{2}^{2} s_{1} s_{2}^{n}, s_{2}^{m} s_{1} s_{2}^{2} s_{1} s_{2}^{3} s_{1} \quad(m, n=1,2, \cdots, 5) .
$$

*Quarterly Journal of Mathematics, vol. 30 (1898), p. 258.

† Hamilon, Philosophioal Magazine, vol. 12 (1856), p. 446. 
In the following generalization it will be convenient to use a group which is defined by a very closely related set of equations, viz.,

$$
s_{1}^{4}=1, \quad s_{2}^{5}=s_{1}^{2}, \quad\left(s_{1} s_{2}\right)^{3}=1 .
$$

Since the icosahedral group does not involve any operators of order 4 the order of the present group is not less than 120. That it is exactly of this order follows from the fact that each of its operators may be written in one of the following forms :

$$
s_{2}^{m}, s_{2}^{m} s_{1} s_{2}^{n}, s_{2}^{m} s_{1} s_{2}^{2} s_{1} s_{2}^{n}, s_{2}^{m} s_{1} s_{2}^{2} s_{1} s_{2}^{3} s_{1} \quad(m=1,2, \cdots, 10 ; n=1,2, \cdots, 5) .
$$

The reductions to these forms follow directly from those of the icosahedral group, since any product involving powers of $s_{1}$ and $s_{2}$ may be written as a power of $s_{2}$ multiplied by a product in which only the first power of $s_{1}$ and the first four powers of $s_{3}$ occur; for $s_{1}^{2}$ and $s_{2}^{5}$ are invariant under $\left\{s_{1}, s_{2}\right\}$.

This group $\left(G_{120}\right)$ of order 120 is well known as an imprimitive group of degree $24 *$ and it seems therefore unnecessary to give an existence proof here. It may be observed that it does not include the icosahedral group, but this group is its group of cogredient isomorphisms. It will be found that the groups whose two generating operators satisfy the generalization of the icosahedral group given by the equations

$$
s_{1}^{2}=s_{2}^{5}, \quad\left(s_{1} s_{2}\right)^{3}=1
$$

are merely direct products of a cyclic group whose order divides 21 and one of the two groups given above. As a first step in this proof, we consider the product of two operators of order two which have a common square, viz., $s_{1}$ and $s_{2}^{-4} s_{1} s_{2}^{4}$. The following equations result directly from the two given relations between $s_{1}$ and $s_{2}$ :

$$
\begin{aligned}
& \left(s_{2}^{-4} s_{1} s_{2}^{4} s_{1}^{-1}\right)^{2}=\left(s_{2}^{2} s_{1}^{-1} s_{2}^{11}\right)^{2}=s_{2}^{2} s_{1}^{-1} s_{2}^{13} s_{1}^{-1} s_{2}^{11} \\
& \left(s_{2}^{-4} s_{1} s_{2}^{4} s_{1}^{-1}\right)^{5}=s_{2}^{2}\left(s_{1}^{-1} s_{2}^{13}\right)^{5} s_{2}^{-2}=s_{2}^{2}\left(s_{1} s_{2}^{3}\right)^{5} s_{2}^{23}=s_{2}^{105} .
\end{aligned}
$$

Since $s_{1}$ transforms $s_{2}^{105}$ into its inverse $\dagger$ and is commutative with it, the order of $s_{2}$ cannot exceed 210. If this order is 210 the two operators $s_{1}^{21}, s_{2}^{21}$ generate the given group of order 120 since $\left(s_{1}^{21} s_{2}^{21}\right)^{3}=s_{2}^{210}\left(s_{1} s_{2}\right)^{3}=1$. Hence $\left\{s_{1}, s_{2}\right\}$ contains the group generated by $s_{2}^{10}$ and this group of order 120 as invariant subgroups having only the identity in common. It is therefore the direct product of these two subgroups, and each of its operators is represented once and only once by one of the following expressions :

$$
s_{2}^{m}, s_{2}^{m} s_{1} s_{2}^{n}, s_{2}^{m} s_{1} s_{2}^{2} s_{1} s_{2}^{n}, s_{2}^{m} s_{1} s_{2}^{2} s_{1} s_{2}^{3} s_{1} \quad(m=1,2, \cdots, 210 ; n=1,2, \cdots, 5) .
$$

\footnotetext{
* It is also the compound perfect group of lowest possible order. Cf. Amerioan Journal of Mathematics, vol. 20 (1898), p. 277.

† Arohiv der Mathematik und Physik, vol. 9 (1895), p. 6.
} 
The cyclic group generated by $s_{2}^{5}$ is composed of the invariant operators of this group and the corresponding quotient group is the icosahedral group. This group of order 2520 may be regarded as the generalization of the icosahedral group which is defined by the equations $s_{1}^{2}=s_{2}^{5}$ and $\left(s_{1} s_{2}\right)^{3}=1$. The other groups whose two generators satisfy these conditions must also satisfy an additional condition, viz., that the order of $s_{2}$ is less than 210. If the order of $s_{2}$ is either 70 or 30 it still follows that $\left(s_{2}^{21} s_{2}^{21}\right)^{3}=1$ and hence the corresponding $\left\{s_{1}, s_{2}\right\}$ is the direct product of the given group of order 120 and either the cyclic group of order 7 or the cyclic group of order 3 .

Finally when the order of $s_{2}$ is 105,35 or 15 , the two operators $s_{1}^{21}, s_{2}^{21}$ generate the icosahedral group and $\left\{s_{1}, s_{2}\right\}$ is the direct product of this group and the cyclic groups of orders $21,7,3$, respectively. If we include the icosahedral group and the given group of order 120, there are exactly eight groups whose two generators satisfy the equations $s_{1}^{2}=s_{2}^{5},\left(s_{1} s_{2}\right)^{3}=1$. In each case the operator of the group is represented once and only once by one of the following forms :

$$
s_{2}^{m}, s_{2}^{m} s_{1} s_{2}^{n}, s_{2}^{m} s_{1} s_{2}^{2} s_{1} s_{2}^{n}, s_{2}^{m} s_{1} s_{2}^{2} s_{1} s_{2}^{3} s_{1} \quad\left(m=1,2, \cdots, \text { order of } s_{2} ; n=1,2, \cdots, 5\right) \text {. }
$$

It may be added that the existence of the given direct products could have been seen directly, since, in the definition of the icosahedron group by means of the equations $s_{1}^{2}=1, s_{2}^{5}=1,\left(s_{1} s_{2}\right)^{3}=1$, it is clearly possible to select an independent operator of order 21 and multiply $s_{1}, s_{2}$, respectively, by its first and its thirteenth powers. The resulting products will satisfy the equations of the generalized icosahedral group. The above proof may therefore be regarded as merely establishing that there are no other such groups.

The two other possible similar generalizations of the icosahedron group can readily be reduced to the preceding one. We shall first consider the case when

$$
s_{1}^{3}=s_{2}^{5}, \quad\left(s_{1} s_{2}\right)^{2}=1 .
$$

To exhibit the connection with the preceding case we let $t_{1}=s_{1} s_{2}$ and $t_{2}=s_{2}^{-1}$. Hence we have to consider $\left\{t_{1}, t_{2}\right\}$ where

$$
t_{1}^{2}=1, \quad t_{2}^{5}=\left(t_{1} t_{2}\right)^{-3} \text {. }
$$

To obtain an upper limit for the order of $t_{2}$ we may use the product $t_{2}^{-4} t_{1} t_{2}^{4} t_{1}$. Since $t_{2}^{5}$ is invariant under $\left\{t_{1}, t_{2}\right\} \equiv\left\{s_{1}, s_{2}\right\}$, it is possible to write the given product in a more compact form as follows: From $t_{2}^{-1} t_{1} t_{2}^{-1} t_{1} t_{2}^{-1} t_{1}=t_{2}^{5}$ it results that $t_{2}^{-4} t_{1} t_{2}^{4} t_{1}=t_{2}^{2} t_{1} t_{2} \cdot t_{2}^{5}$. Hence it results that $\left(t_{2}^{-4} t_{1} t_{2}^{4} t_{1}\right)^{n}=t_{2}^{2}\left(t_{1} t_{2}^{3}\right)^{n} t_{2}^{5 n-2}$. If we write the equation $t_{2}^{-1} t_{1} t_{2}^{-1} t_{1} t_{2}^{-1} t_{1}=t_{2}^{5}$ in the form $t_{1} t_{2}^{3}=t_{2} t_{1} t_{2} t_{1} t_{2}^{4} \cdot t_{2}^{5}$ it is clear that $\left(t_{1} t_{2}^{3}\right)^{n}=t_{2} t_{1} t_{2}^{n} t_{1} t_{2}^{-1} \cdot t^{10 n}$. Letting $n=5$ in these equations we arrive at the equation

$$
\left(t_{2}^{-4} t_{1} t_{2}^{4} t_{1}\right)^{5}=t_{2}^{80}
$$


As $t_{1}$ is commutative with $t_{2}^{80}$ and also transforms it into its inverse, the order of $t_{2}$ is a divisor of 160 . We shall now prove that it cannot be 160 . If $t_{2}$ were of order $160 t_{1} t_{2}$ would be of order 96 . The group generated by $t_{2}^{16}$ and $\left(t_{1} t_{2}\right)^{16}=t_{1} t_{2} \cdot t_{2}^{-25}$ is the same as the one generated by $t_{2}^{16}$ and $t_{1} t_{2} \cdot t_{2}^{-25} \cdot t_{2}^{80}=t_{1} t_{2} \cdot t_{2}^{55}$. As the last operator is of order 3 and as $t_{1} t_{2}^{56} \cdot t_{2}^{-16}=t_{1} t_{2}^{40}$ is of order 4 we are dealing with a group whose two generators $s_{1}, s_{2}$ satisfy the equations $s_{1}^{2}=s_{2}^{5}$, $\left(s_{1} s_{2}\right)^{3}=1, s_{1}^{4}=1$. This is the group of order 120 whose properties were considered above. The cyclic group generated by $t_{2}^{15}$ is invariant under this group and with it generates $\left\{t_{1}, t_{2}\right\}$. This is impossible, since $t_{1}$ would correspond to an operator of order 4 in the $(k, 1)$ isomorphism between $\left\{t_{1}, t_{2}\right\}$ and the given group of order 120 . The order of $s_{2}$ must therefore divide 80 .

It is now easy to prove that there are exactly five groups whose two generators $s_{1}, s_{2}$ satisfy the equations $s_{1}^{3}=s_{2}^{5},\left(s_{1} s_{2}\right)^{2}=1$. They are the direct products of the icosahedron group and the cyclic group of order $2^{\alpha}, \alpha=0,1,2,3,4$. The proof of this theorem follows very readily from the fact that the order of $s_{2}$ divides 80 . Hence $\left\{s_{1}^{16}, s_{2}^{16}\right\}$ is the icosahedron group, since $\left(s_{1}^{16} s_{2}^{16}\right)^{2}=\left(s_{1} s_{2}\right)^{2}=1$ and $s_{1}^{16}, s_{2}^{16}$ are of orders 3 and 5 respectively. As $\left\{s_{1}, s_{2}\right\}$ contains the cyclic group generated by $s_{2}^{5}$ and the icosahedron group as invariant subgroups having only the identity in common and the order of $\left\{s_{1}, s_{2}\right\}$ cannot exceed the product of the orders of this invariant subgroup, the proof is complete.

It remains only to consider the groups whose two generators satisfy the equations

$$
s_{1}^{2}=s_{2}^{3}, \quad\left(s_{1} s_{2}\right)^{5}=1 .
$$

It will be convenient to use the auxiliary symbols $t_{1}=s_{1}, t_{2}=s_{1} s_{2}$. Proceeding as before we have

Hence

$$
\left(t_{2}^{-4} t_{1} t_{2}^{4} t_{1}^{-1}\right)^{n}=t_{2}^{2}\left(t_{1} t_{2}^{3}\right)^{n} t_{2}^{3} s_{2}^{-12 n}, \quad\left(t_{1} t_{2}^{3}\right)^{n}=t_{2} t_{1} t_{2}^{n} t_{1} t_{2}^{4} \cdot s_{2}^{-6 n-3} .
$$

$$
\left(t_{2}^{-4} t_{1} t_{2}^{4} t_{1}^{-1}\right)^{5}=s_{2}^{-90} \text {. }
$$

Moreover, $\left(t_{2}^{-2} t_{1} t_{2}^{2} t_{1}^{-1}\right)^{3}=s_{2}^{42}\left(t_{2}^{2} t_{1}\right)^{5} t_{2}^{-8}=s_{2}^{75}$. Since $t_{1}$ transforms both $s_{2}^{-80}$ and $s_{2}^{75}$ into their inverses and is commutative with each of them, the order of $s_{2}$ divides 30 .

If the order of $s_{2}$ is 30 that of $s_{1}$ is 20 . In this case $s_{1}^{5}, s_{2}^{10}$ generate the group $G_{120}$ considered above; for $\left(s_{1}^{5} s_{2}^{10}\right)^{5}=s_{2}^{15}$ and the orders of $s_{1}^{5}, s_{2}^{10}$ are 4 and 3 respectively. As $s_{1}^{4}$ is invariant under this group and has only the identity in common with it and as $\left\{s_{1}^{4}, s_{1}^{5}, s_{2}^{10}\right\} \equiv\left\{s_{1}, s_{2}\right\}$ it follows that the latter is the direct product of $G_{120}$ and the cyclic group of order 5 . When the order of $\delta_{2}$ is 15 that of $s_{1}$ is 10 and $s_{1}^{5}, s_{2}^{10}$ generate the icosahedron group. In this case $s_{1}^{5} s_{2}^{10}=s_{2}^{15} s_{1} s_{2}=s_{1} s_{2}$. When the order of $s_{2}$ is either 6 or $3,\left\{s_{1}, s_{2}\right\}$ is either the given group of order 120 or the icosahedron group. Hence the theorem: 
There are exactly four groups whose two generators $s_{1}, s_{2}$ satisfy the equations $s_{1}^{2}=s_{2}^{3},\left(s_{1} s_{2}\right)^{5}=1$. They are the icosahedron group, $G_{120}$, and the direct products of these groups and the cyclic group of order 5 .

Combining the results of this section, we conclude that there are fourteen distinct groups which are generated by two operators satisfying one of the three immediate generalizations of the defining relations of the icosahedron group. The generators of the icosahedron group satisfy each of these generalized definitions, those of $G_{120}$ satisfy two of them, while those of each of the other twelve groups determined above satisfy only one of these sets of conditions. The simple defining relations and the close contact with the simple group of lowest composite order make these fourteen groups of peculiar interest. 